\title{
Reptiles en corredores biológicos y mortalidad por atropellamiento vehicular en Barbas-Bremen, Quindío, Colombia
}

\author{
Daniel Felipe López-Herrera', Mateo León-Yusti ${ }^{1}$, Stefanny Caroline Guevara-Molina², Fernando Vargas-Salinas ${ }^{2, *}$ \\ ${ }^{1}$ Programa de Biología, Universidad del Quindío, Armenia, Colombia \\ ${ }^{2}$ Grupo de Investigación en Evolución, Ecología y Conservación (EECO). \\ Programa de Biología, Universidad del Quindío, Armenia, Colombia
}

\begin{abstract}
Resumen
Los corredores biológicos de Barbas-Bremen son una estrategia de conservación implementada en el departamento del Quindío, Colombia, con el objetivo de reestablecer la conectividad entre poblaciones bióticas presentes en los dos fragmentos de bosque más grandes de la región. No obstante, pocos estudios han evaluado la efectividad de estos corredores como hábitat para especies de fauna local y como facilitadores de dispersión de individuos entre las reservas naturales de Barbas-Bremen. En 18 salidas de campo realizadas entre julio de 2014 y marzo de 2015 caracterizamos la fauna de reptiles en tres corredores biológicos y se evaluó si la mortalidad de reptiles por atropellamiento vehicular en una carretera que atraviesa dichos corredores biológicos está correlacionada con la distancia hasta ellos. Utilizamos encuentros visuales libres y trampas de caída para muestrear la riqueza de reptiles en cada corredor biológico y áreas de pastizal adyacentes; los individuos atropellados los registramos durante 34 caminatas en la carretera. Encontramos 42 individuos pertenecientes a 16 especies; los reptiles en los corredores biológicos tienden a ser arbóreos mientras que los de pastizales tienden a ser terrestres. No se observaron lagartos atropellados en carretera y no hubo relación entre el número de atropellos (64 serpientes, 9 especies) y la distancia al corredor biológico más cercano. En general, los corredores biológicos de Barbas-Bremen ofrecen un hábitat boscoso para numerosas especies de reptiles que probablemente no podrían subsistir en la matriz de pastizales que predomina en el área de estudio; sin embargo, se requieren más estudios para establecer si dichos corredores biológicos favorecen el flujo de individuos entre BarbasBremen y cómo la carretera que los atraviesa afectaría este proceso. (c) 2016. Acad. Colomb. Cienc. Ex. Fis. Nat.
\end{abstract}

Palabras clave: Ofidios; Lagartos; Conservación; Atropellamiento.

Reptiles in biological corridors and roadkills in Barbas-Bremen, Quindío, Colombia

\begin{abstract}
The biological corridors of Barbas-Bremen are a conservation strategy implemented in the department of Quindío, Colombia, in order to reestablish connectivity between biotic populations in the two largest forest fragments in the region. However, very few studies have evaluated the effectiveness of those corridors as habitat for local wildlife and as facilitators of dispersal of individuals between the natural reserves of Barbas-Bremen. Between July 2014 and March 2015 we performed 18 fieldtrips to characterize the reptile fauna in three biological corridors to test whether mortality of reptiles on a road that crosses the biological corridors is correlated with the distance to them. We used visual sampling and pitfall traps to sample the richness of reptiles in each biological corridor and adjacent grassland; roadkills were recorded during 34 walks on the road. We found 42 reptiles belonging to 16 species; reptiles in biological corridors tend to be arboreal, the species in grassland tend to be terrestrial. There was no relationship between the number of roadkills (64 snakes, 9 species) and the distance to the nearest biological corridor. We did not observe lizards killed on the road. In general, the Barbas-Bremen biological corridors offer a forested habitat to numerous species of reptiles that probably could not survive in the grassland that prevails in the study area; however, more research is required to determine whether these biological corridors encourage the flow of individuals between Barbas-Bremen, and the role that the road there plays in this process. (C) 2016. Acad. Colomb. Cienc. Ex. Fis. Nat.
\end{abstract}

Key words: Snakes; Lizards; Conservation; Roadkills

\section{Introducción}

La destrucción y la consecuente fragmentación de hábitats naturales en Colombia es cada vez más alta (Correa, Ruiz, Arévalo, 2006), lo que sugiere un efecto negativo cada vez mayor en las poblaciones silvestres. Los Andes Colombianos albergan cerca del 70 \% de la población humana en el país, lo cual ha conllevado a una deforestación de 82.661 ha/ año y la modificación de más del $80 \%$ de los bosques montanos y pre-montanos en la región (Franco \& Ruiz,

\section{*Correspondencia:}

Fernando Vargas-Salinas, fvargas@uniquindio.edu.co

Recibido: 8 de febrero de 2016

Aceptado: 2 de agosto de 2016 
2014). Lo anterior ha creado en los Andes colombianos un paisaje compuesto por potreros, cultivos agrícolas, franjas de vegetación riparia y parches de bosque secundario (Kattan, et. al., 2004; Cabrera, et al. 2011). El efecto en pérdida de biodiversidad producida por la deforestación en los Andes colombianos es particularmente preocupante, pues es la región con mayor riqueza de especies en el país, muchas de ellas endémicas y con rangos de distribución altamente restringidos (Myers, et. al., 2000; Forero-Medina \& Joppa, 2010; Graham, et al. 2014).

Una de las estrategias para mitigar la pérdida de diversidad biológica por la destrucción y fragmentación de hábitats naturales es la implementación de corredores biológicos. Los corredores biológicos como estrategia de conservación se han implementado en diversos ecosistemas del planeta desde principios de los años 70 con resultados positivos en grupos como anfibios y reptiles (Reyes-Puig, et al. 2012), aves (Stanley, et. al., 1977; Kroodsma, 1982; Betts, et al. 2014), mamíferos (Simberloff, et. al., 1992; Hodgson, et. al., 2009; LaPoint, et. al., 2013) e insectos (Valle, et. al., 2013). Sin embargo, el establecimiento de corredores biológicos también ha sido controversial, ya que su implementación es costosa y en muchos casos no se evalúa su eficacia en la conectividad de poblaciones (Simberloff, et al. 1992; Lapoint, et al. 2013); además, los corredores biológicos pueden favorecer la dispersión de enfermedades, plagas, depredadores o la propagación de incendios forestales (Beier \& Noss, 1998; Bennet, 1998; Ryall \& Fahrig, 2006; Resasco, et al. 2014; Atobe, et. al., 2014).

Se conocen más de 80 iniciativas de corredores biológicos en Sur América, y en ese contexto, Colombia es uno de los países con mayor número de proyectos, 17 hasta hoy (Graham \& Kalemani, 2006). El proyecto de los corredores biológicos de Barbas-Bremen en el departamento del Quindío, Andes centrales de Colombia, ha sido calificado como uno de los mejores a nivel internacional, pues integra áreas protegidas, promueve la conectividad funcional, conserva la biodiversidad y presenta una planificación del uso de la tierra como un medio para alcanzar los objetivos de conservación (Renjifo, 2004; Graham \& Kalemani, 2006). La efectividad de los corredores de Barbas-Bremen como conectores entre la biota del Cañón del río Barbas y la Reserva Forestal de Bremen se ha evaluado en las aves y los insectos (Marín-Gómez, et al. 2009; Ortiz-Movliav, 2014; Montealegre-Talero, 2014); sin embargo, los resultados en estos estudios no pueden generalizarse, ya que la respuesta a la fragmentación de hábitat y a las estrategias de manejo y conectividad varían entre grupos biológicos (Schelhas \& Greenberg, 1996; Pardini, et al. 2009).

Los reptiles no han sido tenidos en cuenta ni durante la planeación de los corredores biológicos de Barbas-Bremen ni después de su implementación. Su inclusión en las estrategias de manejo y conservación es importante, pues son vertebrados que hacen parte integral del flujo de nutrientes ya sea como depredadores o presas (Reagan \& Waide, 1996;
Greene, 1997; Lynch, 2012). A pesar de su importancia ecológica y de que cerca del $20 \%$ de las especies de reptiles están en riesgo de extinción, se han estudiado poco desde el punto de vista de la biología de la conservación (Gibbons, et al., 2000, Bohm, et al., 2013). En este estudio registramos los reptiles presentes en los corredores biológicos de Barbas-Bremen y sus áreas adyacentes. Además, dado que los corredores biológicos son atravesados por una carretera con alta tasa de atropellamiento de reptiles (Quintero-Ángel, et al., 2012), nos propusimos examinar si los individuos que utilizan los corredores biológicos son especialmente susceptibles a morir atropellados, ya sea porque intentan cruzar la carretera para alcanzar los corredores en la margen contraria de la vía, o porque utilizan el asfalto de la carretera como una superficie cálida para su termorregulación (Ashley \& Robinson, 1996; Shine, et al., 2004).

\section{Materiales y métodos}

\section{Área de estudio}

El área de estudio se encuentra ubicada sobre la vertiente occidental de la Cordillera Central de Colombia; la vegetación nativa en el área es de bosque muy húmedo montano y premontano (Franco \& Bravo, 2005), pero actualmente los bosques remanentes están ubicados en una matriz donde predominan pastizales utilizados para ganadería, pinos y eucaliptos, y monocultivos de mora, sábila y aguacate, entre otros (Etter, 1998; Renjifo, 2001). El Cañón del río Barbas ( $04^{\circ} 42^{\prime} \mathrm{N}$ y $75^{\circ} 38^{\prime} \mathrm{O}$; que en adelante se denominará Barbas) y la Reserva Forestal Bremen (04ㄴㅇ' $\mathrm{N}$ y $75^{\circ} 37^{\prime} \mathrm{O}$; que en adelante se denominará Bremen) están ubicados entre los 1.500 y los $2.600 \mathrm{msnm}$, en los departamentos de Quindío y Risaralda (Renjifo, 2004). El área combinada de Barbas-Bremen tiene cerca de 1.600 ha, siendo el área de bosque con mayor tamaño de toda la región (GómezPosada, et al., 2006; Pérez, 2007).

En el área de estudio el Instituto de Investigaciones Biológicas Alexander von Humboldt estableció cinco corredores biológicos (Colibríes, Pavas, Laureles, Monos y otro al cual no se le asignó nombre) para favorecer la conectividad de fauna y flora entre las poblaciones en Barbas-Bremen (Lozano, et al., 2006; Vargas, et al., 2009). Estos corredores biológicos están localizados en el municipio de Filandia (Quindío) el cual exhibe temperaturas que oscilan entre los 12 y los $27^{\circ} \mathrm{C}$ y una precipitación media anual de 2.739 $\mathrm{mm}$; los meses de julio y agosto son los más secos mientras que, los meses de marzo-mayo y octubre-diciembre son los más lluviosos (Arcila, et al., 2011). El ancho actual de los corredores biológicos varía de 28 a 289 m (MontealegreTalero, 2014) y consisten en bosques lineales de especies vegetales nativas (arbóreas y arbustivas) que fueron establecidos hace más de diez años (Lozano, et al., 2006). Los corredores biológicos en el área de estudio son atravesados por una carretera de aproximadamente ocho metros de ancho que conecta al pueblo de Filandia con la Autopista del Café, la cual va de Armenia a Pereira (Lozano, et al. 2006). 


\section{Fase de campo}

Para nuestro estudio seleccionamos tres corredores biológicos (Colibríes, Pavas y Monos) con base en su continuidad desde Barbas hasta Bremen y su accesibilidad debido a la topografía, la logística y los permisos en predios privados. Para conocer las especies de reptiles que utilizan estos tres corredores biológicos hicimos muestreos de encuentros visuales libres (Visual encounter surveying, VES; Crump \& Scott, 1994; Rueda-Almonacid, et al., 2006) en áreas del corredor biológico tanto cerca $( \pm 15 \mathrm{~m})$ como lejos (>400 m) de la carretera que lo atraviesa. Para examinar y comparar la fauna de reptiles en los corredores biológicos con la que se encuentra en áreas abiertas adyacentes, se utilizó este mismo tipo de muestreo en los pastizales que rodean los corredores biológicos tanto en áreas cercanas a la carretera como lejos de ella.

Entre julio de 2014 y marzo de 2015 se hicieron 18 salidas de campo, con una duración promedio de tres a cuatro días cada una. La metodología de muestreo consistió en caminatas diurnas (9:00 am - 3:00 pm) y nocturnas (7:00 pm - 12:00 pm) durante las cuales buscamos individuos sobre ramas, arbustos y en cavidades de troncos. Además, utilizamos tridentes para remover la materia orgánica del suelo (para el caso de los corredores biológicos) y para rastrillar los pastizales más altos. Como un complemento a la búsqueda visual de reptiles, entre octubre de 2014 y marzo de 2015 utilizamos trampas de caída distribuidas en los corredores biológicos y en áreas abiertas cerca y lejos de la carretera. Estas trampas consistieron en cubetas plásticas de $50 \mathrm{~cm}$ de profundidad y $30 \mathrm{~cm}$ de diámetro enterradas con su abertura a ras del suelo; para llevar a los individuos hacia la trampa de caída pusimos una banda de polipropileno de $8 \mathrm{~m}$ de largo y $40 \mathrm{~cm}$ de alto, enterrada a $10 \mathrm{~cm}$ de profundidad que atravesaba la abertura de la cubeta. Estas trampas se revisaron diariamente durante los lapsos del trabajo en campo; al finalizar el muestreo se cubrían las cubetas para evitar la caída de individuos.

Para examinar si los reptiles que utilizan los corredores biológicos son particularmente propensos a morir por atropellamiento, registramos el número de reptiles atropellados y su distancia hasta el borde del corredor biológico más cercano. Para este análisis tuvimos en cuenta todos los corredores biológicos en el área de estudio. Los registros de cadáveres en carretera se hicieron durante 34 caminatas en 3,8 km de la carretera realizadas por dos personas entre agosto de 2014 y marzo de 2015. Las caminatas se hicieron a diferentes horas del día; a cada cadáver se le hizo una fotografía, se registró su posición con un GPS Garmin Oregón 550t que posteriormente se removió para evitar un doble conteo. La identificación taxonómica de los individuos atropellados se hizo con base en las especies registradas en los corredores biológicos y pastizales aledaños, y su posible presencia en el área (Peters \& Orejas-Miranda, 1970; Colorado \& Ramírez, 2001; Quintero-Ángel, et al., 2012, Vanegas-Guerrero, et al., 2015; Vanegas-Guerrero, et al., 2016).

\section{Análisis de datos}

Para saber qué tan completos fueron nuestros muestreos en los corredores biológicos, los pastizales y la carretera, se analizó la cobertura de muestreo (Chao \& Jost, 2012) en la plataforma virtual de iNEXT (Hsieh, et al., 2015). Este análisis estima la proporción del número total de individuos en una comunidad que pertenece a una especie registrada en el muestreo (Chao \& Jost, 2012). Además, el análisis de cobertura de muestreo corrige sesgos existentes en metodologías tradicionales que se basan en curvas de acumulación de especies para comparar la diversidad entre sitios después de estandarizar por tamaño de muestra (principio de duplicidad sensu Chao \& Jost 2012 y Chao, et al. 2014). El recambio en la composición de especies entre los corredores biológicos (diversidad beta) y los pastizales aledaños, se examinó con el índice de similitud de Chao-Jaccard (Chao, et al., 2005, 2006), el cual se calculó en Estimates 9,0 (Colwell, et al. 2012) y se graficó en un dendrograma utilizando PAST 3,0 (Hammer, et al., 2013). mediante la prueba de correlación de Pearson se analizó si la frecuencia de atropellos de reptiles por segmentos de carretera de $50 \mathrm{~m}$ seguía un patrón aleatorio o no con respecto a la distancia al corredor biológico más cercano; para este análisis calculamos la distancia al corredor biológico desde el punto medio de cada segmento de carretera.

\section{Resultados}

Se registraron 16 especies de reptiles (seis lagartos y diez serpientes, Tabla 1 y Figura 1). Seis especies son endémicas para Colombia pero ninguna está incluida en categoría de amenaza (Castaño-Mora, 2002). Siete especies son de actividad arbórea y nueve son terrestres o fosoriales. Los reptiles en los corredores biológicos tienden a ser especies arbóreas, mientras que las especies en los pastizales son de actividad principalmente terrestre (Tabla 1).

El muestreo de reptiles en los corredores biológicos y los pastizales alcanzó una cobertura de muestreo de 0,89 y 0,67, respectivamente (Figura 2). A estos valores de cobertura de muestreo se asoció una riqueza media de doce especies en corredores biológicos y cuatro en pastizales. Ninguna de las especies registradas en los corredores biológicos se registró en los pastizales (Tabla 1) lo cual, es congruente con los resultados del análisis de similitud (Figura 3).

En 34 caminatas a lo largo de la carretera no se observó ningún lagarto atropellado, sin embargo, registramos 64 cadáveres de serpientes pertenecientes a por lo menos nueve especies, siete géneros y tres familias; 12 individuos no pudieron ser identificados debido a su estado avanzado de descomposición (Tabla 2). Con base en las especies identificadas, se logró una cobertura de muestreo equivalente a 0,97 para la carretera (Figura 2C). La serpiente Atractus cf. melanogaster Werner 1916 fue la especie con mayor número de registros (28 individuos atropellados, $45 \%$ de los 
Tabla 1. Riqueza de especies, abundancia de individuos y patrones de actividad para reptiles registrados en el área de estudio: corredores biológicos de Filandia (Colibríes, Pavas, Monos) y pastizales aledaños, Quindío, Andes Centrales de Colombia. El área abierta (pastizal) aledaña al corredor biológico de Pavas no pudo ser evaluada por acceso restringido a propiedad privada. Las especies señaladas con asterisco $(*)$ son endémicas en Colombia.

\begin{tabular}{|c|c|c|c|c|c|c|c|}
\hline \multirow[t]{2}{*}{ TAXA } & \multicolumn{2}{|c|}{ Hábito } & \multicolumn{2}{|c|}{ Colibríes } & \multirow{2}{*}{$\begin{array}{c}\text { Pavas } \\
\text { Corredor }\end{array}$} & \multicolumn{2}{|c|}{ Monos } \\
\hline & Terrestre & Arbóreo & Corredor & Pastizal & & Corredor & Pastizal \\
\hline \multicolumn{8}{|l|}{ ORDEN SQUAMATA } \\
\hline \multicolumn{8}{|l|}{ SUBORDEN LACERTILIA } \\
\hline \multicolumn{8}{|l|}{ Dactyloidae } \\
\hline Anolis ventrimaculatus* & & $\bullet$ & & & 4 & 5 & \\
\hline Anolis antonii* & & $\bullet$ & & & 3 & & \\
\hline Anolis eulaemus & & $\bullet$ & & & 2 & & \\
\hline \multicolumn{8}{|l|}{ Gymnopthalmidae } \\
\hline Anadia rhombiphera & $\bullet$ & & 2 & & 1 & 1 & \\
\hline Gymnopthalmus sp & $\bullet$ & & 1 & & 1 & & \\
\hline \multicolumn{8}{|l|}{ Sphaerodactylidae } \\
\hline Lepidoblepharis duolepis* & $\bullet$ & & & & 1 & & \\
\hline \multicolumn{8}{|l|}{ SUBORDEN SERPENTES } \\
\hline \multicolumn{8}{|l|}{ Dipsadidae } \\
\hline Erythrolamprus epinephelus & $\bullet$ & & & 3 & & & 2 \\
\hline Leptodeira annulata & & $\bullet$ & & & 1 & & \\
\hline Dipsas pratti & & $\bullet$ & & & 1 & 1 & \\
\hline Atractus cf. melanogaster* & $\bullet$ & & & 1 & & & \\
\hline Atractus titanicus* & $\bullet$ & & & 1 & & & \\
\hline \multicolumn{8}{|l|}{ Colubridae } \\
\hline Tantilla melanocephala & $\bullet$ & & & & & & 1 \\
\hline Chironius exoletus & & $\bullet$ & & & 1 & & \\
\hline \multicolumn{8}{|l|}{ Leptotyphlopidae } \\
\hline Trilepida joshuai* & $\bullet$ & & 1 & & 2 & 3 & \\
\hline \multicolumn{8}{|l|}{ Elapidae } \\
\hline Micrurus mipartitus & $\bullet$ & & & & & 1 & \\
\hline \multicolumn{8}{|l|}{ Viperidae } \\
\hline Bothriechis schlegelii & & $\bullet$ & & & & 2 & \\
\hline
\end{tabular}

registros). Las serpientes atropelladas se registraron entre 0 y 696 metros de distancia del borde del corredor biológico más cercano; la mayoría (94,1 \%) de individuos atropellados corresponden a siete especies de áreas abiertas (Figura 4). Encontramos una relación inversa entre la frecuencia de atropellos y la distancia al corredor biológico más cercano $(\mathrm{r}=$-0.527, $p=0,044)$; sin embargo, cuando hicimos el mismo análisis sin incluir el valor extremo de 18 serpientes observadas adyacente a los corredores biológicos (Figura 4), dicha relación desapareció ( $r=-0,234, p=0,117)$.

\section{Discusión}

Nuestros resultados corroboran que los corredores biológicos de Barbas-Bremen son utilizados como hábitat por diversas especies de reptiles (lagartos, serpientes) y que estas tienden a ser diferentes de aquellas en pastizales. La baja cobertura de muestreo, especialmente para las áreas de pastizal, podría haber incrementado los cálculos de diversidad beta entre los corredores biológicos y sus alrededores (Kattan, et al. 2006). Sin embargo, aunque algunas especies pudiesen estar presentes en ambos tipos de hábitat y no haber sido registrado en los muestreos, creemos que el patrón que sugiere nuestro análisis de similitud es real y no un artificio debido al número de individuos en la muestra. El alto recambio de especies entre los corredores biológicos y los pastizales adyacentes sería el reflejo de diferencias abruptas en microclima, estructura vegetal y de diferencias en requerimientos específicos de recursos tales como el sitio de percha, ítems alimenticios y riesgo de depredación (Carvajal-Cogollo \& Urbina- 
Cardona, 2015; Lion, et al., 2016). Los lagartos Anolis ventrimaculatus Boulenger 1911 y A. eulaemus Boulenger 1908, por ejemplo, duermen en perchas arbóreas que no están presentes en áreas de pastizal, los pequeños lagartos del género Sphaerodactylus son comunes en microhábitats húmedos que se dan entre la hojarasca y troncos caídos que

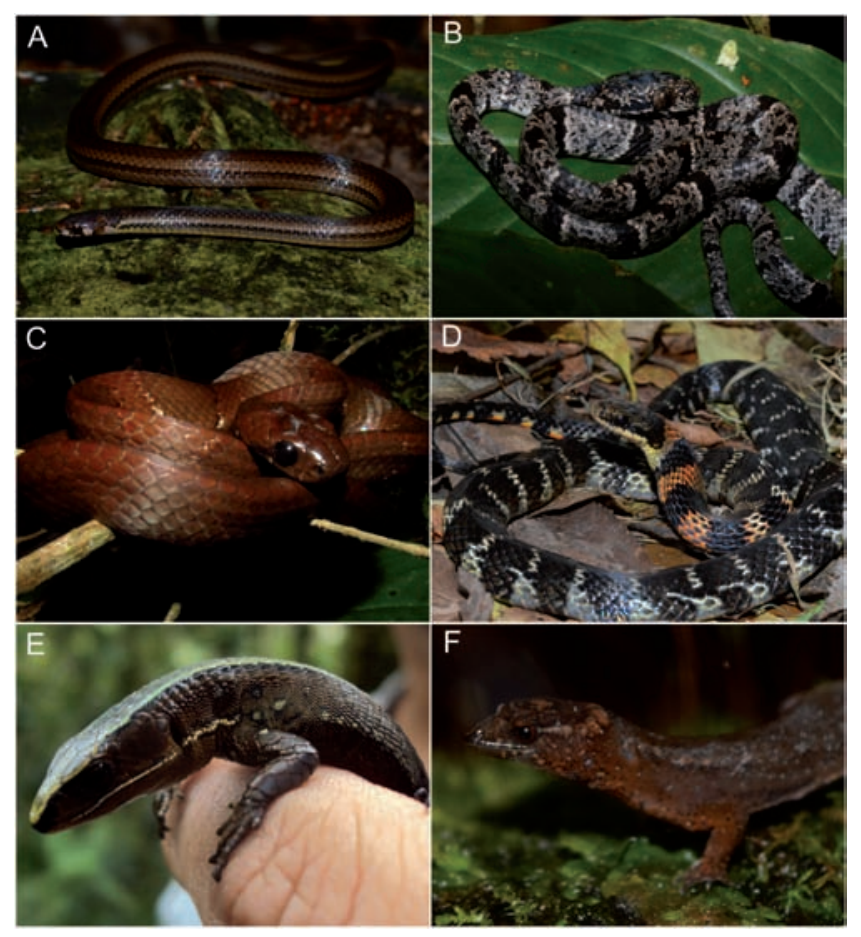

Figura 1. Imagen de algunos de los reptiles registrados en los corredores biológicos, áreas de pastizal y carretera. A) Tantilla melanocephala, B) Sibon nebulatus, C) Dipsas pratti, D) Erythrolamprus epinephelus, E) Anadia rhombifera, F) Lepidoblepharis duolepis. Fotografías: Fernando Vargas-Salinas (A, B, D), Caroline Guevara-Molina (C, F) y Daniel López-Herrera (E). predominan en áreas con cobertura arbórea (Savage, 2002), mientras que la serpiente Bothriechis schlegelii Berthold 1986 frecuenta ramas y hojas donde se alimenta de aves, ranas y pequeños mamíferos (Campbell \& Lamar, 2004).

No se puede determinar si las especies de reptiles presentes en los corredores biológicos provienen o no de las reservas de Barbas-Bremen, pues es factible que provengan de remanentes boscosos presentes en el paisaje antes y durante la implementación del proyecto de conservación. En cualquier caso, es de esperar que los reptiles hayan colonizado los corredores biológicos a medida que los procesos de sucesión vegetal avanzaron. Diversos estudios han documentado que con el avance de un proceso de sucesión vegetal hacia el establecimiento de áreas boscosas, la fauna va recolonizando a medida que se incrementa la complejidad vegetal y la disponibilidad de recursos (Johnston \& Odum, 1956; Huey, et al., 1989; Goode, et al. 2005; Hernández-Ordoñez, et al., 2015). Por ejemplo, microhábitats húmedos en troncos caídos, hoyos y grietas son de vital importancia para la recolonización de especies de reptiles terrestres (Goode, et al., 2005), mientras que las especies arbóreas requieren del establecimiento de una cobertura vegetal leñosa y la disponibilidad previa de ítems tales como insectos y pequeños vertebrados (Ríos-López \& Aide, 2007).

La ausencia de lagartos atropellados podría deberse a que su tamaño corporal pequeño hace que los cadáveres no persistan mucho tiempo sobre el asfalto de la carretera y, por lo tanto, nuestros registros estarían sesgados. Esta posibilidad encuentra sustento en que otros estudios de fauna atropellada en Colombia usualmente no registran lagartos a menos que sean especies de tamaño corporal relativamente grande (e.g. Iguana iguana) (Argotte \& Monsalvo, 2002; Payan, et al., 2013; De la Ossa \& De la Ossa, 2013; De la Ossa \& Galván-Guevara, 2015). Sin embargo, en nuestro caso descartamos esta posibilidad, ya
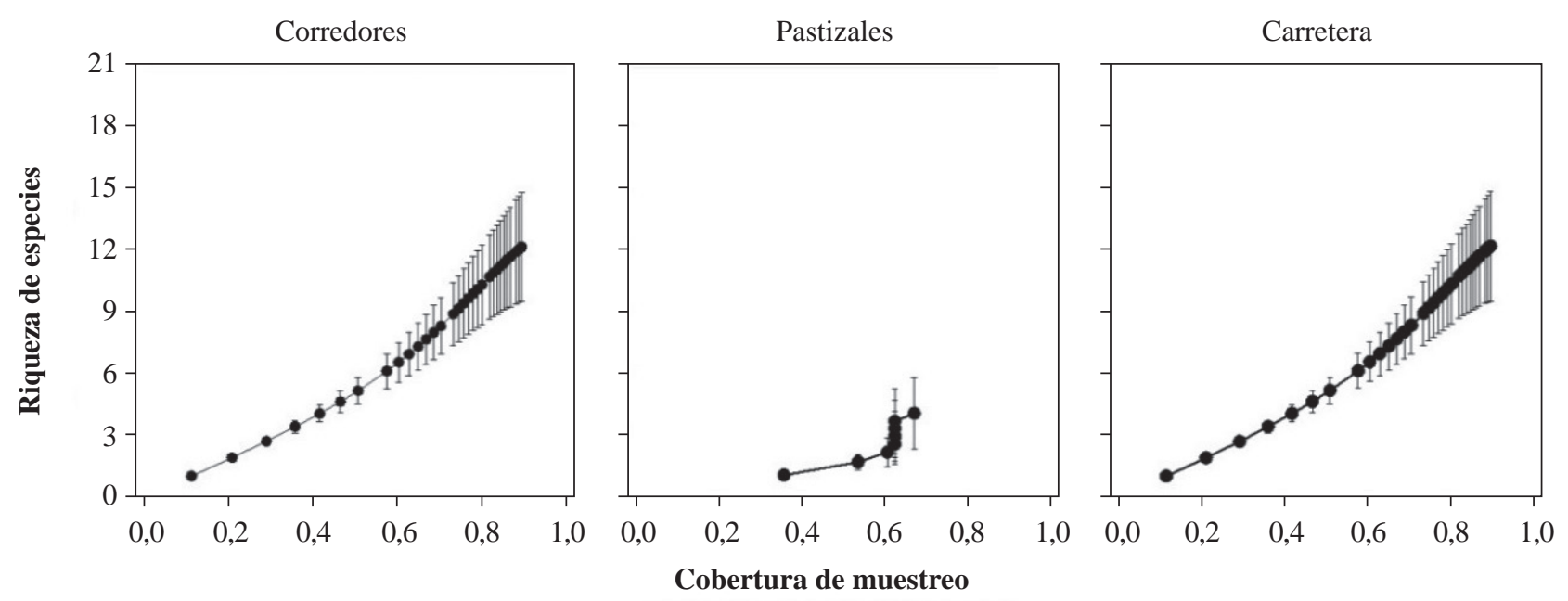

Figura 2. Curvas de cobertura de muestreo para reptiles en corredores biológicos, pastizales y carretera. En los pastizales no se observaron lagartos y en la carretera solo se registraron serpientes atropelladas. 
Rev. Acad. Colomb. Cienc. Ex. Fis. Nat. 40(156):484-493, julio-septiembre de 2016 doi: http://dx.doi.org/10.18257/raccefyn.334

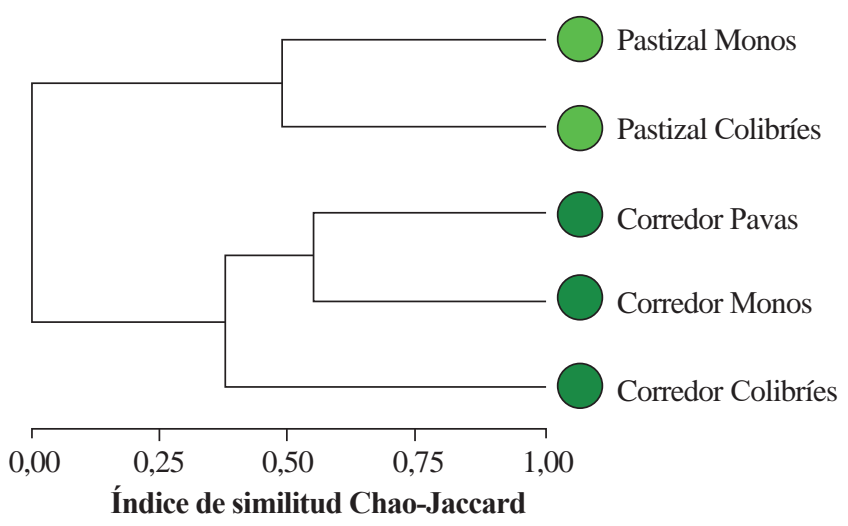

Figura 3. Nivel de similitud de especies de reptiles en tres corredores biológicos y dos áreas de pastizal aledañas a ellos en el área de estudio (vía entre Autopista del Café y municipio de Filandia, Andes centrales de Colombia)

Tabla 2. Reptiles atropellados en 3,8 kilómetros del tramo de la carretera entre la autopista del Café y el municipio de Filandia, en Quindío, Andes Centrales de Colombia

\begin{tabular}{lrrrrrrrr}
\hline & \multicolumn{2}{c}{$\mathbf{2 0 1 4}$} & & \multicolumn{2}{c}{2015} \\
\hline TAXA & Ago & Oct & Nov & Ene & Feb & Marzo & Total \\
\hline Dipsadidae & & & & & & & \\
\hline Atractus cf. melanogaster & 7 & 4 & 4 & 4 & 3 & 7 & 29 \\
\hline Atractus titanicus & 1 & 1 & 0 & 1 & 0 & 0 & 3 \\
\hline Atractus sp. & 0 & 0 & 0 & 0 & 1 & 0 & 1 \\
\hline Dipsas pratti & 0 & 1 & 0 & 1 & 0 & 0 & 2 \\
\hline Erythrolamprus epinephelus & 1 & 1 & 0 & 0 & 1 & 0 & 3 \\
\hline Sibon nebulata & 1 & 0 & 0 & 0 & 0 & 0 & 1 \\
\hline Colubridae & & & & & & & \\
\hline Tantilla melanocephala & 2 & 0 & 2 & 3 & 1 & 2 & 10 \\
\hline Urotheca cf. decipiens & 1 & 0 & 0 & 1 & 0 & 0 & 2 \\
\hline Viperidae & & & & & & & \\
\hline Bothriechis schlegelii & 1 & 0 & 0 & 0 & 0 & 0 & 1 \\
\hline No identificados & 5 & 2 & 1 & 1 & 1 & 2 & 12 \\
\hline Total & $\mathbf{1 9}$ & $\mathbf{9}$ & $\mathbf{7}$ & $\mathbf{1 1}$ & $\mathbf{7}$ & $\mathbf{1 1}$ & $\mathbf{6 4}$ \\
\hline
\end{tabular}

que por lo menos tres especies de lagartos (A. eulaemus, $A$. ventrimaculatus, Anadia rhombifera) tienen igual o mayor masa corporal que algunas de las serpientes registradas en la carretera (Atractus cf. melanogaster, Tantilla melanocephala Linnaeus 1758). Creemos que la ausencia de lagartos atropellados en la carretera se debe a varias posibilidades no excluyentes entre sí y que en un futuro deben ser evaluadas para entender mejor los procesos que explican las diferencias entre las tasas de atropellos de diferentes grupos faunísticos. Primero, entre los lagartos los atropellos de los individuos y especies que se inhiben al cruzar la vía habrían sido más frecuentes (Forman, et al. 2003); segundo, los lagartos de áreas boscosas podrían evitar áreas abiertas donde pueden

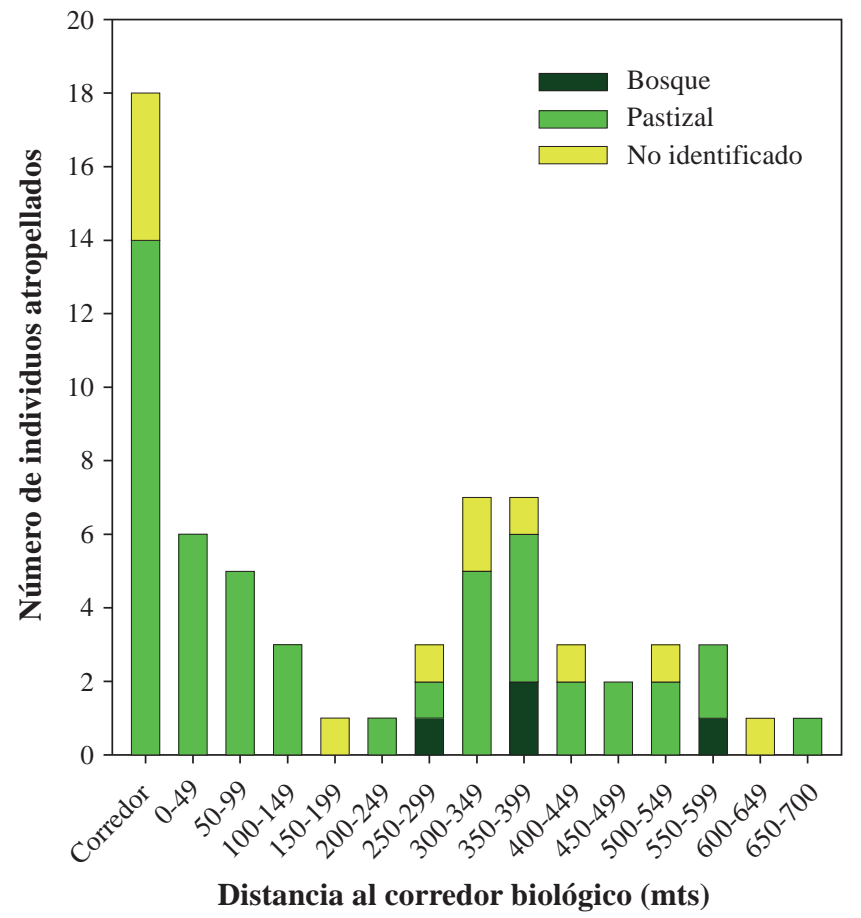

Figura 4. Frecuencia de serpientes atropelladas en segmentos de carretera con respecto a rangos de distancia al corredor biológico más cercano y tipo de hábitat predominante para cada especie

ser fácilmente depredados por aves (Roughgarden, 1995; Losos, 2009); tercero, contrario a las serpientes, las especies de lagartos en el área de estudio no utilizan el asfalto de la carretera como superficie para termorregularse, y cuarto, los lagartos no asumen una postura estática y defensiva ante los vehículos como se ha reportado en serpientes (Andrews \& Gibbons, 2005; Shine, et al. 2004).

La relación inversa entre el número de serpientes atropelladas en la carretera y la distancia al corredor biológico no es concluyente; es decir, las especies que utilizan los corredores biológicos no son especialmente susceptibles a morir atropelladas. Concluimos esto, en primera instancia por que dicha relación está altamente influenciada por un registro extremo de serpientes (18 individuos) en segmentos de la carretera adyacentes a los corredores; segundo, la mayoría de dichas serpientes son de especies asociadas a áreas abiertas y no a áreas boscosas. La mayoría de serpientes en los corredores biológicos son de hábito arbóreo y dicho comportamiento posiblemente reduce su tendencia a cruzar áreas abiertas y carentes de vegetación. Nuestros resultados concuerdan con los reportados por QuinteroÁngel, et al. (2012), quienes encontraron que las serpientes atropelladas en esta misma área de estudio fueron en su gran mayoría especies asociadas a pastizales. Este patrón sugiere que las especies de hábitos terrestres tienden a acercarse y cruzar la carretera con mayor frecuencia que las especies con hábitos arbóreos o arbustivos (Jochimsen, et al., 2014). Una tendencia similar se ha registrado en 
mamíferos, lagartos y anfibios (Fahrig, et al. 1995; Ashley \& Robinson, 1996; Argotte \& Monsalvo, 2002; Baskaran \& Boominathan, 2010).

En general, el alto número de serpientes atropelladas en el Neotrópico sugiere que las carreteras representan una amenaza para la conservación de estos vertebrados (Gibbons \& Semlitsch, 1987; Ashley \& Robinson, 1996; Shine, et al., 2004; Andrews \& Gibbons, 2008; Hartmann, et al., 2011; Vargas-Salinas, et al., 2011; Quintero-Ángel, et al., 2012). No obstante, un alto número de individuos atropellados no necesariamente significa un impacto negativo a nivel poblacional (Fahrig \& Rytwinski, 2013; Fahrig, et al., 2013); por lo tanto, es necesario hacer observaciones más prolongadas antes de concluir si las poblaciones de serpientes en el área de estudio estarían experimentando una reducción (Reading, et al., 2010).

La reforestación que implicó el establecimiento de los corredores biológicos en el área de estudio pudo incrementar la cobertura boscosa disponible para algunas especies de reptiles que requieren de este tipo de hábitat. En este sentido, los corredores de Barbas-Bremen podrían estar jugando un rol similar al de pequeños fragmentos boscosos en la conservación de reptiles (Turner \& Corlett 1996; Lion, et al., 2016). Por otro lado, nuestros resultados no permiten corroborar si dichos corredores biológicos están conectando las poblaciones presentes en los fragmentos boscosos de Barbas-Bremen, tal como se ha sugerido para arañas y aves (Marín-Gómez, et al., 2009; MontealegreTalero 2014; Ortiz-Movliav 2014).

\section{Conclusiones}

Los corredores biológicos de Barbas-Bremen sirven de hábitat a especies de reptiles que probablemente no podrían subsistir en la matriz de pastizales y cultivos que predominan en el paisaje del área de estudio, pero aún no se puede inferir si hay flujo de individuos entre las reservas naturales de Barbas-Bremen. La carretera en el área de estudio crea una alta mortalidad de serpientes, pero la mayoría de los individuos atropellados son de especies de pastizales y no de aquellas registradas en los corredores biológicos. En futuros estudios se deben evaluar si los pocos atropellamientos de individuos de especies registradas en los corredores biológicos se debe a que se inhiben de cruzar la carretera o a su gran capacidad para cruzar la vía sin morir en el intento.

\section{Agradecimientos}

Gracias a Sebastián Tabares, Benjamín Rivero, Alejandro Ochoa y Gustavo Arias por su ayuda en el trabajo de campo; al grupo de Herpetología de la Universidad del Quindío (GHUQ) por reconfirmar la identificación de algunos individuos; a Jorge Iván Salazar y Juan Carlos García por facilitar nuestra estadía en campo; a la UMATA y a los residentes del Municipio de Filandia por permitir nuestro ingreso a los corredores biológicos. El Instituto Alexander Von Humboldt permitió la utilización de los datos de reptiles obtenidos bajo la ejecución del proyecto Planeación Ambiental para la Conservación de la Biodiversidad en las áreas operativas de Ecopetrol S.A, en Barbas-Bremen, Filandia-Quindío (2014).

\section{Conflicto de intereses}

Los autores no tienen ningún conflicto de intereses frente a la información presentada en este documento.

\section{Referencias}

Agostinho, A.A., Gómez, L.C., Fernández, D.R., \& Suzuki, H. 2002. Efficiency of fish ladders for neotropical ichthyo fauna. River Research and Applications. 18 (3): 299-306.

Andrews, K.M., \& Gibbons, W.J. 2005. How do highways influence snake movement? behavioral responses to road and vehicles. Copeia. 4: 772-782.

Andrews, K.M., \& Gibbons, W.J. 2008. Ecological effects of roads on amphibians and reptiles: A literature review. Herpetological Conservation. 3: 121-143.

Arcila, P.J., Ramírez, B.V.H., \& Jaramillo, R.A. 2011. Patrones de distribución de la lluvia en la zona cafetera. Cenicafé. 5-11.

Argotte, S.D., \& Monsalvo, M.J.D. 2002. Incidencia de la carretera Barranquilla-Ciénaga sobre la mortalidad de vertebrados y su relación con el medio ecológico en la vía Parque Isla de Salamanca, Magdalena, Colombia (tesis de pregrado). Universidad del Atlántico, Barranquilla, Colombia.

Ashley, E.P., \& Robinson, J.T. 1996. Road mortality of amphibians, reptiles and other wildlife on the long point causeway. The Canadian Field-Naturalist. 110 (3): 403-412.

Atobe, T., Osada, Y., Takeda, H., Kuroe, M., \& Miyashita, T. 2014. Habitat connectivity and resident shared predators determine the impact of invasive bullfrogs on native frogs in farm ponds. Proceedings of the Royal Society B: Biological Sciences. 281 (1786): 1-10.

Baskaran, N., \& Boominathan, D. 2010. Road kills of animals by highway traffic in the tropical forest of mudumalai tiger reserve, southern India. Journal of Threatened Taxa. 2 (3): 753-759.

Beier, P., \& Noss, R.F. 1998. Do habitat corridors provide connectivity?. Conservation Biology. 12 (6): 1241-1252.

Bennett, A.F. 1998. Enlazando el Paisaje: el papel de los corredores biológicos y la conectividad en la conservación de la vida silvestre. IUCN, Gland, Suiza y Cambridge.

Betts, M.G., Fahrig, L., Hadley, A.S., Halstead, K.E., Bowman, J., Robinson, W. D., \& Lindenmayer, D.B. 2014. A speciescentered approach for uncovering generalities in organism responses to habitat loss and fragmentation. Ecography. 37 (6): 517-527.

Bohm, M., Collen, B., Baillie, J.E.M., Bowles, P., Chanson, J., Cox, N., \& Rhodin, A.G. 2013. The conservation status of world's reptiles. Biological Conservation. 157: 372-385.

Cabrera, E., Vargas, D.M., Galindo, G., García, M.C., Ordóñez, M.F., Vergara, L.K., Pachecho, A.M., Rubiano, J.C., \& Giraldo, P. 2011. Memoria técnica de la cuantificación de la deforestación histórica nacional - escala gruesa y fina. IDEAM. Bogotá, D.C., Colombia.

Campbell, J.A., \& W.W. Lamar. 2004. The venomous reptiles of the western hemisphere. Comstock Publishing, Cornell University. Ithaca, New York, Estados Unidos. 475 pp. 
Carvajal-Cogollo, J.E. \& J.N. Urbina-Cardona. 2015. Ecological grouping and edge effects in tropical dry forest: reptilemicroenvironment relationships. Biodiversity and Conservation. 24 (5): 1109-1130.

Castaño-Mora, O.V. 2002. Libro rojo de reptiles de Colombia. La serie libro rojos de especies amenazadas de Colombia. (Castaño-Mora, O.V. ed.). Bogotá, D.C. Instituto de Ciencias Naturales, Universidad Nacional de Colombia.

Chao, A., Chazdon, R.L., Colwell, R.K., \& Shen, T. 2005. A new statistical approach for assessing similarity of species composition with incidence and abundance data. Ecology. 8: $148-159$

Chao, A., Chazdon, R.L., Colwell, R.K., \& Shen, T. 2006. Abundance-based similarity indices and their estimation when there are unseen species in samples. Biometrics. 62 (2): 361-371.

Chao, A., \& Jost, L. 2012. Coverage-based rarefaction and extrapolation: standardizing samples by completeness rather than size. Ecology. 93 (12): 230- 250.

Chao, A., Gotelli, N.J., Hsieh, T.C., Sander, E.L., Ma, K.H., Colwell, R.K., \& Ellison, A.M. 2014. Rarefaction and extrapolation with Hill numbers: a framework for sampling and estimation in species diversity studies. Ecological Monographs. 84 (1): 45-67.

Colorado, A.M., \& Ramírez, W. 2001. Diversidad y distribución de las comunidades de serpientes en 11 localidades del Quindío (tesis de pregrado). Universidad del Quindío, Colombia.

Colwell, R.K., Chao, A., Gotelli, N.J., Lin, S., Mao, C.X., Chazdon, R.L., \& Longino, J.T. 2012. Models and estimators linking individual-based and sample-based rarefaction, extrapolation and comparison of assemblages. Journal of Plant Ecology. 5 (1): 3-21.

Correa, H., Ruiz, S., \& Arévalo, L. 2006. Plan de Acción en la biodiversidad de la cuenca del Orinoco-Colombia 20052015. Propuesta técnica. Corporinoquia, Cormacarena, IAvH, Unitrópico, Fundación Omacha, Fundación Horizonte Verde, Universidad Javeriana, Unillanos, WWF-Colombia. Bogotá D.C.

Crump, M.L., \& Scott, N.J. 1994. Visual Encounter Surveys. En: W. Heyer, M.A. Donnelly, R.A. McDiarmid, L.C. Hayec, M.C. Foster (eds). Measuring and Monitoring Biological Diversity. (pp. 84-92). Washington DC: Smithsonian Institution Press.

De La Ossa, N.O., \& De La Ossa, V.J. 2013. Fauna silvestre atropellada en dos vías principales que rodean los Montes de María, Sucre, Colombia. Revista Colombiana de Ciencia Animal. 5 (1): 158-164.

De La Ossa, V.J. \& Galván-Guevara, S. 2015. Registro de Mortalidad de Fauna Silvestre por Colisión Vehicular en la Carretera Tolú Viejo - Ciénaga la Caimanera, Sucre Colombia. Biota Colombiana. 16 (1): 67-77.

Etter, A. 1998. Mapa general de ecosistemas de Colombia (Escala 1: 2'000.000). Instituto Alexander von Humboldt. Universidad Javeriana. Bogotá, Colombia.

Fahrig, L., Pedlar, J.H., Pope, S.E., Taylor, P.D., \& Wegner, J.F. 1995. Effect of road traffic on amphibian density. Biological Conservation. 73 (3): 177-182.

Fahrig, L., \& Rytwinski, T. 2013. Effects of roads on animal abundance: an empirical review and synthesis. Ecology and Society. 14 (1): 21.
Forero-Medina, G., \& Joppa, L. 2010. Representation of global and national conservation priorities by Colombia's protected area network. PlosONE. 5 (10): 3210.

Forman, R.T..T., Sperling, D., Bissonette, J., Clevenger, A., Cutshall, C., Dale, V., Fahrig, L., France, R., Goldman, C., Heanue, K., Jones, J., Swanson, F., Turrentine, T., \& Winter, T. 2003. Road Ecology: Science and Solutions. Island Press, Washington, DC. 481 pp.

Franco, A.M., \& Bravo, G. 2005. Áreas Importantes para la Conservación de las Aves en Colombia. En: K. Boyla, A. Estrada (eds). Áreas Importantes para la Conservación de las Aves en los Andes Tropicales: sitios prioritarios para la conservación de la biodiversidad. Quito, Ecuador. Serie de Conservación de Birdlife.

Franco, V.L., \& Ruiz, J.P. 2014. V Informe Nacional de Biodiversidad de Colombia, ante el convenio de diversidad biológica. Instituto Alexander von Humboldt. Bogotá D.C. Colombia.

Gibbons, J.W., \& Semlitsch, R.D. 1987. Activity patterns. En: R.A. Seigel, J.T. Collins, S.S. Novak (eds). Snakes: Ecology and Evolutionary Biology. MacMillan, Nueva York. 529 pp.

Gibbons, J.W., Scott, D.E., Ryan, J.T., Buhlmann, K.A., Tuberville, T.D., Metts, B.S., \& Winne, C.T. 2000. The global decline of Reptiles, Deja Vu Amphibians. Bioscience. 50: 653-666.

Gómez-Posada, C., Giraldo, C.G., Zulima, I.A., \& Londoño, J. 2006. Evaluación de la densidad poblacional del mono aullador rojo (Alouatta seniculus) en bosques del eje cafetero. Fundación Ecoandina/Wildlife Conservation Society, Cali, Colombia.

Goode, M.J., Horrace, W.C., Sredl, M.J., \& Howland, J.M. 2005. Habitat destruction by collectors associated with decreased abundance of rockdwelling lizards. Biological Conservation. 125 (1): 47-54.

Graham, B., \& Kalemani, J.M. 2006. Review of Experience with Ecological Networks, Corridors and Buffer Zones. Secretariat of the Convention on Biological Diversity.

Graham, C.H., Carnaval, A.C., Cadena, C.D., Zamudio, K.R., Roberts, T.E., Parra, J.L., \& Schneider, C.J. 2014. The origin and maintenance of montane diversity: integrating evolutionary and ecological processes. Ecography. 37: 711-719.

Greene, H.W. 1997. Snakes, the evolution of mystery in nature. California, USA. Editorial University of California press. $351 \mathrm{pp}$.

Hammer, O., Harper, D.A.T., \& Ryan, P.D. 2013. PAST: Paleontological statistics software package for education and data analysis. PalaeoE. 4 (1): 9.

Hartmann, P.A., Hartmann, M.T., \& Martins, M. 2011. Snake road mortality in a protected area in the Atlantic Forest of southeastern Brazil. South American Journal of Herpetology. 6 (1): 35-42.

Hernández-Ordóñez, O., Urbina-Cardona, N., \& MartínezRamos, M. 2015. Recovery of amphibian and reptile assemblages during old-field succession of tropical rain forests. Biotropica. 47 (3): 377-388.

Hodgson, J.A., Thomas, C.D., Wintle, B.A., \& Moilanen, A. 2009. Climate change, connectivity and conservation decision making: back to basics. Journal of Applied. Ecology. 46 (5): 964-969.

Hsieh, T., Ma, K., \& Chao, C. 2015. iNEXT: An R package for interpolation and extrapolation in measuring species diversity. 
Huey, R.B., Peterson, C.R., Arnold, S.J., \& Porter, W.P. 1989. Hot rocks and not-so-hot rocks: retreat-site selection by garter snake and its thermal consequences. Ecology. 70: 931-944.

Jochimsen, D.M., Peterson, C.R., \& Harmon, L.J. 2014. Influence of ecology and landscape on snake road mortality in a sagebrush-steppe ecosystem. Animal Conservation. 17 (6): 583-592.

Johnston, D.W., \& Odum, E.P. 1956. Breeding bird populations in relation to plant succession on the piedmont of Georgia. Ecology. 37: 50-62.

Kattan, G.H., Franco, P., Rojas, V., \& Morales, G. 2004. Biological diversification in a complex region: a spatial analysis of faunistic diversity and biogeographic of the Andes of Colombia. Journal of Biogeography. 31 (11): 1829-1839.

Kattan, G.H., Franco, P., Saavedra-Rodríguez, C.A., Valderrama, C., Rojas, V., Osorio, D., \& Martínez, J. 2006. Spatial components of bird diversity in the Andes of Colombia: Implications for designing a regional reserve system. Conservation Biology. 20: 1203-1211.

Kroodsma, R.L. 1982. Bird community ecology on power-line corridors in East Tennessee. Biological Conservation. 23 (2): 79-94.

LaPoint, S., Gallery, P., Wikelski, M., \& Kays, R. 2013. Animal behavior, cost-based corridor models, and real corridors. Journal of Landscape Ecology. 28 (8): 1615-1630.

Lion, M.B., Garda, A.A., Santana, D.J., \& Fonseca, C.R. 2016. The conservation value of small fragments for Atlantic forest reptiles. Biotropica. 48 (2): 265-275.

Losos, J.B. 2009. Lizards in an evolutionary tree: ecology and adaptive radiation of anoles. London, England. Editorial University of California Press. 528 pp.

Lozano, F.H., Vargas, A.M., Vargas, W., Jiménez, E., Mendoza JE, Caicedo, P., Aristizabal, S.L., Ramírez, D.P., Murillo, X., \& Ríos, C.I. 2006. Modelo de manejo sostenible de paisajes rurales para la conservación de la biodiversidad en la región andina colombiana. Línea de investigación conservación de la biodiversidad en paisajes rurales Instituto Alexander Von Humboldt. Colombia.

Lynch, J.D. 2012. El contexto de las serpientes de Colombia con un análisis de las amenazas en contra de su conservación. Revista de la Academia Colombiana de Ciencias Exactas, Físicas y Naturales. 36 (140): 435-449.

Montealegre-Talero, C. 2014. Uso de hábitat y rangos de hogar de especies focales de aves en un paisaje andino fragmentado (tesis de pregrado). Pontificia Universidad Javeriana. Bogotá D.C., Colombia.

Marín-Gómez, O.H., Banguera, N.Y., \& Carmona, P.J. 2009. "Monitoreo" de la avifauna amenazada del Aica Barbas-Bremen y evolución de la población de pava caucana (Penelope perspicax) en el cañón del río Barbas. Fundación Ornitológica del Quindío. Instituto Alexander Von Humboldt.

Myers, N., Mittermeier, R.A., Mittermeier, C.G., Fonseca, G.A.B., \& Kent, J. 2000. Biodiversity hots pots for conservation priorities. Nature. 403: 853-858.

Ortiz-Movliav, C. 2014. Evaluación de la diversidad de arañas como indicadoras de la efectividad de las estrategias de restauración implementadas en el corredor biológico Barbas-Bremen, Filandia (Quindío-Colombia) (tesis de pregrado). Universidad Icesi. Cali, Colombia.
Pardini, R., Fariaa, D., Accacio, G.M., Laps, R.R., Mariano-Neto, E., Paciencia, M.L.B., Dixon, M., \& Baumgarten, J. 2009. The challenge of maintaining Atlantic forest biodiversity: A multi-taxa conservation assessment of specialist and generalist species in an agro-forestry mosaic in southern Bahia. Biological Conservation. 142: 1178-1190.

Payan, E., Soto, C., Diaz-Pulido, A., Benítez, A., \& Hernández, A. 2013. Wildlife road crossing and mortality: lessons for wildlife friendly road design in Colombia. Proceedings of the 2013 International Conference on Ecology and Transportation (ICOET).

Pérez, A. 2007. Aplicación del enfoque ecosistémico en latinoamérica. Commission on Ecosystem Management. Bogotá, D.C., Colombia. Unión Internacional Para La Conservación de la Naturaleza.

Peters, J.A., \& Orejas-Miranda, B. 1970. Catalogue of the neotropical squamata: Part I. Snakes. Bulletin-United States National Museum. 297 pp.

Quintero-Ángel, A., Osorio-Domínguez, S., Vargas-Salinas, F. \& Saavedra-Rodríguez, C.A. 2012. Roadkill rate of snakes in a disturbed landscape of central Andes of Colombia. Herpetology Notes. 5: 99-105.

Reading, C.J., Luiselli, L.M., Akani, G.C., Bonnet, X., Amori, G., Ballouard, J.M., \& Rugiero, L. 2010. Are snake populations in widespread decline?. Biology Letters. 11 (11): $1-4$.

Reagan, D.P., \& Waide, R.B. 1996. The food web of a tropical forest. Chicago USA. Editorial The University of Chicago Press. 616 pp.

Renjifo, L.M. 2001. Effect of natural and anthropogenic landscape matrices on the abundance of sub Andean bird species. Ecological Applications. 11 (1): 14-31.

Renjifo, L.M. 2004. Aplicación del enfoque ecosistémico a la gestión de corredores en América del Sur. Quito, Ecuador. Unión internacional para la conservación de la naturaleza en América del Sur.

Resasco, J., Haddad, N.M., Orrock, J.L., Shoemaker, D., Brudvig, L.A., Damschem, E.I., \& Levey, D.J. 2014. Landscape corridors can increase invasion by an exotic species and reduce diversity of native species. Ecology. 95 (8): 2033-2039.

Reyes-Puig, J.P., \& Yánez-Muñoz, M.H. 2012. Una nueva especie de Pristimantis (Anura: Craugastoridae) del corredor ecológico llangantes-sangay, Andes de Ecuador. Papéis Avulsos do Departamento de Zoologia. 52 (6): 81- 91.

Ríos-López, N., \& Aide, T.M. 2007. Herpetofaunal dynamics during secondary succession. Herpetologica. 63 (1): 35-50.

Roughgarden, J. 1995. Anolis lizards of the Caribbean. New York, USA. Editorial Oxford University Press. 226 pp.

Rueda-Almonacid, J.V., Castro, F., \& Cortéz, C. 2006 Técnicas para el inventario y muestreo de anfibios: una compilación. En: Ángulo, A., Rueda-Almonacid, J.V., RodríguezMahecha, J.V., \& La Marca, E. (eds.). Técnicas de inventario y monitoreo para los anfibios de la región tropical andina. (Panamericana ed.). Bogotá DC, Colombia. 300 pp.

Ryall, K.K., \& Fahrig, L. 2006. Response of predators to loss and fragmentation of prey habitat: a review of theory. Ecology. 87 (5): 1086-1093.

Savage, J.M. 2002. The amphibians and reptiles of Costa Rica: a herpetofauna between two continents, between two seas. London, England. Editorial University of Chicago Press. $934 \mathrm{pp}$. 
Rev. Acad. Colomb. Cienc. Ex. Fis. Nat. 40(156):484-493, julio-septiembre de 2016 doi: http://dx.doi.org/10.18257/raccefyn.334

Schelhas, J., \& Greenberg, R.S. 1996. Forest patches in tropical landscapes. Washington D.C., USA. Editorial Island Press. 337 pp.

Shine, R., LemasteR, M., Wall, M., Langkilde, T., \& Mason, R. 2004. Why did the snake cross the road? Effects of roads on movement and location of mates by garter snakes (Thamnophis sirtalis parietalis). Ecology and Society. 9 (1): 9-21.

Simberloff, D., Farr, J.A., Cox, J., \& Mehlman, D.W. 1992. Movement corridors: conservation bargains or poor investments. Conservation Biology. 6 (4): 493-504.

Stanley, H.A., Shugart, J., Herman, H., \& Kathleen, M. 1977. The effect of transmission-line corridors on bird populations. The American Midland Naturalist. 97 (1): 216-221.

Turner, I.M., \& Corlett, R.T. 1996. The conservation value of small, isolated fragments of lowland tropical rain forest. Trends in Ecology and Evolution. 11: 330-333.

Vanegas-Guerrero, J., Gómez-Hoyos, D.A., Gómez-López, C.M., \& Londoño-Guarnizo, C.A. 2015. Reptilia, Sauria, Gymnophthalmidae, Anadia rhombifera (Günther, 1859): distribution extensión and first records from Quindío department, Colombia. Check List. 11 (1): 1512.

Vanegas-Guerrero, J., Fernández, C.A., Buitrago-González, W.E., \& Vargas-Salinas, F. 2016. Urban Remnant Forests: Are they important for herpetofaunal conservation in the Central Andes of Colombia?. Herpetological Review. 47 (2): 180-185.

Vargas, W., Renjifo, L.M., Aristizábal, S.L., Lozano-Zambrano, F.H., Vargas, A.M., \& Ramírez, D.P. 2009. Herramientas de manejo para la conservación de biodiversidad en paisajes rurales. Capítulo 4: Diseño de la estrategia de conservación en el paisaje rural (Fase II), Instituto Alexander Von Humboldt. Bogotá D.C., Colombia.

Vargas-Salinas, F., Delgado-Ospina, I, López-Aranda, F. 2011. Mortalidad por atropello vehicular y distribución de anfibios y reptiles en un bosque sub andino en el occidente de Colombia. Caldasia. 33 (1): 121-138.

Valle, I.C., Buss, D.F., \& Baptista, D.F. 2013. The influence of connectivity in forest patches, and riparian vegetation width on stream macroinvertebrate fauna. Brazilian Journal of Biology. 73 (2): 231-238. 